\title{
Detection and characterization of long-pulse low-velocity impact damage in plastic bonded explosives
}

\author{
Pengwan Chen ${ }^{\mathrm{a}, *}$, Fenglei Huang ${ }^{\mathrm{a}}$, Kaida Dai ${ }^{\mathrm{a}}$, Yansheng Ding ${ }^{\mathrm{b}}$ \\ ${ }^{a}$ National Key Laboratory of Explosion and Safety Science, Beijing Institute of Technology, Beijing 100081, China \\ ${ }^{\mathrm{b}}$ Institute of Mechanics, Chinese Academy of Sciences, Beijing 100080, China
}

Received 11 November 2003; received in revised form 21 January 2004; accepted 27 January 2004

\begin{abstract}
Damage not only degrades the mechanical properties of explosives, but also influences the shock sensitivity, combustion and even detonation behavior of explosives. The study of impact damage is crucial in the vulnerability evaluation of explosives. A long-pulse low-velocity gas gun with a gas buffer was developed and used to induce impact damage in a hot pressed plastic bonded explosive. Various methods were used to detect and characterize the impact damage of the explosive. The microstructure was examined by use of polarized light microscopy. Fractal analysis of the micrographs was conducted by use of box counting method. The correlation between the fractal dimensions and microstructures was analyzed. Ultrasonic testing was conducted using a pulse through-transmission method to obtain the ultrasonic velocity and ultrasonic attenuation. Spectra analyses were carried out for recorded ultrasonic signals using fast Fourier transform. The correlations between the impact damage and ultrasonic parameters including ultrasonic velocities and attenuation coefficients were also analyzed. To quantitatively assess the impact induced explosive crystal fractures, particle size distribution analyses of explosive crystals were conducted by using a thorough etching technique, in which the explosives samples were soaked in a solution for enough time that the binder was totally removed. Impact induces a large extent of explosive crystal fractures and a large number of microcracks. The ultrasonic velocity decreases and attenuation coefficients increase with the presence of impact damage. Both ultrasonic parameters and fractal dimension can be used to quantitatively assess the impact damage of plastic bonded explosives.
\end{abstract}

(C) 2004 Elsevier Ltd. All rights reserved.

Keywords: Plastic bonded explosives; Impact damage; Ultrasonic tests; Microstructure; Particle size distribution

\footnotetext{
*Corresponding author. Tel.: + 86-106-891-2858; fax: +86-106-846-1701.

E-mail address: pwchen@bit.edu.cn (P. Chen).
} 


\section{Introduction}

Plastic bonded explosives (PBX) are highly particle filled composite materials and are widely used in both civil and military applications when high performance is required. It is well recognized that the mechanical properties of composite materials may degrade severely with the presence of damage. Damage not only deteriorates the mechanical properties of explosives, but also influences the sensitivity, combustion and even detonation behavior of explosives. Damaged energetic materials have higher risks of the formation of hot spots, combustion abnormity, shockto-detonation-transition (SDT) and deflagration-to-detonation-transition (DDT) [1,2]. Damaged energetic materials are more vulnerable to external stimuli. Experimental results reveal that delayed detonation (XDT) under low amplitude impact may be associated with the presence of mechanical damage $[3,4]$.

Impact damage of explosives may arise from events such as drops, launching and penetration. The low strengths and risks of combustion and explosion bring additional difficulties to the study of impact damage of explosive materials. Drop weight can be used to induce damage under intermediate level strain rates [5]. The damage produced under high strain rates can be achieved using high-speed projectiles and flying plates [6-8]. However when conventional gas guns and explosive-driven flying plates are used in dynamic loading, they may induce excessive damage and even cause accidental reaction or detonation of explosives. To overcome this, we developed a long-pulse low-velocity gas gun incorporated with a gas buffer and used it to apply dynamic loading.

Damage from impact may not be visible to the naked eye. Various damage modes, such as matrix cracking, interface debonding and particle fractures may be present in impact damaged explosives. The application of damage detection and assessment of composite structures, especially impact damage, is still in its infancy.

Ultrasonic testing (UT) has been used for a long time in nondestructive testing. It is a technique that utilizes changes in energy as a mechanical wave propagates through a medium, to detect flaws. Through spectrum analysis of recorded ultrasonic signals, the master frequency and spectrum area can be obtained. Ultrasonic tests have been used to correlate impact loading and damage extent of composites under low- and high-velocity impact [9-11].

Fractal geometry has become a powerful tool in the analysis of irregular features such as impact damage. Fractal geometry analyses have been conducted on microstructural features such as grain boundaries [12], spalling surfaces of an Al alloy [13]. Fractal dimensions can provide much useful information of the microstructure of materials.

PBXs have a very high content of explosive particles, resulting in a large extent of particle-to-particle contacts. When subjected to compressive stress such as pressing and impact, contact induced crystal fractures are expected to happen. Microscopic examination shows the presence of considerable crystal fractures in pressed PBX and impact damaged PBXs [14,15]. Crystal fractures may cause the variation of particle size distribution and correspondingly the formation of new surfaces, which may increase the risks of shock initiation. However, little work has been done to quantitatively assess the explosive crystal fractures induced by pressing and impact. In this paper, an attempt was made to use a so-called thorough etching technique to characterize the evolution of explosive particle size distribution before and after impact. 


\section{Experiment and analysis}

\subsection{Material}

Hot pressed PBXN-5 was used in the experiments. PBXN-5 contains HMX 94.5-95\% and fluorin rubber $5.0-5.5 \%$ by weight. The molding powder was pressed into a disc sample with a size of $\phi 20 \mathrm{~mm} \times 10 \mathrm{~mm}$. The pressing pressure and temperature were $200 \mathrm{MPa}$ and $100^{\circ} \mathrm{C}$, and the duration of pressing was $1.5 \mathrm{~h}$. The density of the samples is $1.85 \pm 0.06 \mathrm{~g} / \mathrm{cm}^{3}$ and the porosity is $2.5 \pm 0.4 \%$.

\subsection{Long-pulse low-velocity impact tests}

To avoid unexpected excessive damage or even detonation of explosives and better control the damage extent, a long-pulse dynamic loading technique was developed. The technique was developed from conventional low-velocity gas gun. Its principle is to lower the peak stress and extend the duration of dynamic loading by avoiding direct impact. To realize it, a gas buffer was developed and installed on the gun. The stress pulse can be adjusted by filling the buffer with different pressure of gases.

The experiment setup is shown in Fig. 1. An explosive sample is constrained in a steel tube. Two polyethylene cushions are placed between the explosive sample and two steel rods. The diameter and length of projectiles are 56 and $110 \mathrm{~mm}$, respectively. Aluminum projectiles are used, and their weight is $0.72 \mathrm{~kg}$. A stress gauge is mounted on steel rod 1 to record the stress history during impact loading. The projectile firstly impacts the gas buffer, and then acts on the explosive sample via steel rod 2 and a polyethylene cushion. The projectile velocities are measured by two probes placed at the end of the gas gun.

Fig. 2 shows two recorded stress history curves of PBXN-5 samples impacted by an aluminum projectile at a velocity of $75.5 \mathrm{~m} / \mathrm{s}$ with a gas buffer and at a velocity of $71 \mathrm{~m} / \mathrm{s}$ without a gas buffer. The peak stress and duration of stress pulse were about $29.8 \mathrm{MPa}$ and $8 \mathrm{~ms}$, respectively for impact with the gas buffer, compared with $644 \mathrm{MPa}$ and $1 \mathrm{~ms}$ without the gas buffer. It is clearly shown that the presence of the gas buffer noticeably extends the duration and lowers the peak stress of dynamic loading.

After impact, the explosive samples were collected for further examination including microscopic examination, ultrasonic testing and particle size distribution analysis.

\subsection{Microscopic examination and fractal analysis}

Polarized light microscopy (PLM) was used to examine the microstructure of explosive samples. An explosive usually has an exceptionally low toughness. In addition, explosive materials

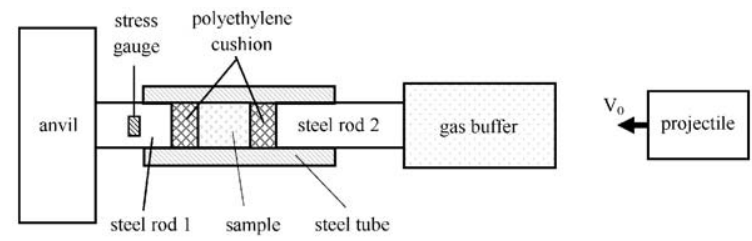

Fig. 1. Experimental setup of dynamic loading. 
have risks of combustion and explosion. These bring difficulties to the preparation of microscopic samples. In our experiment, samples were first ground using standard fine silicon carbide papers (800 grit) to obtain a flat surface. Final polishing was carried out in an automatic polishing machine using $1 \mu \mathrm{m}$ alpha alumina powder, at a load of $50 \mathrm{~g}$, while being lubricated with distilled water. To avoid bringing additional unexpected damage to impact damaged samples in polishing, the samples were first potted in commercial low-viscosity epoxide mounts with traditional amine hardening agent and then cured. To better reveal the details of the microstructure, iso-methyl butyl ketone was selected to etch the surface. The polished and etched samples were directly used for PLM examination. The whole polishing process can be monitored by using a CCD camera. To avoid the accumulation of friction heat and causing fatal accident in the polishing process, continuous distilled water was required to cool the samples. Fig. 3 shows a schematic description of an impact damaged sample. Three representative fields, including near-field (A), mid-field (B) and far-field $(\mathrm{C})$, were chosen for examination.

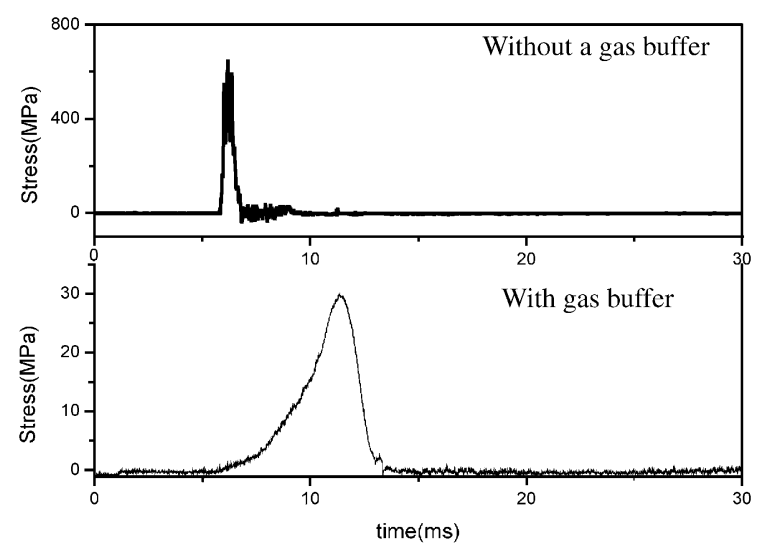

Fig. 2. Typical stress history curves of impact loading with and without the gas buffer.

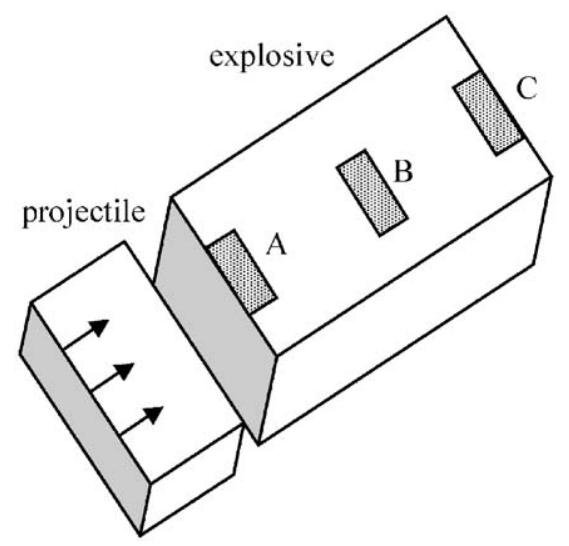

Fig. 3. Schematic of view fields. 
Fractal analyses were based on image processing of the micrographs of explosive samples. The procedure to obtain the fractal dimension of the images was that of box counting [16,17], which consists in the superposition of a series of boxes on top of the image and that they are added if any pixel falls within the box boundaries. The size of the boxes, in pixels, is varied following an exponent 2 progression, i.e. $1 \times 1,2 \times 2,4 \times 4$, etc. The fractal dimension $\left(D_{\mathrm{f}}\right)$ of the image can be computed as [16]

$$
D_{\mathrm{f}}=1+\frac{\log \left(N_{P}\right)}{\log (P)}
$$

where $N_{p}$ is the number of boxes that contains at least one pixel of the image, and $P$ is the size of the box in pixels.

\subsection{Ultrasonic testing}

Ultrasonic examination of the explosive samples was conducted using a pulse throughtransmission method, in which water was used as a coupling medium. The ultrasonic pulse velocity $C_{p}$ was obtained from the measured time delay $\Delta t$ between the trigger signal corresponding to the pulse emission and the first received signal. In ultrasonic attenuation tests, incident signal, reflected signal and transmitted signal were recorded. The attenuation coefficient was calculated as

$$
\alpha=\frac{20}{h} \log \left[\left(\frac{A_{\mathrm{i}}}{A_{\mathrm{t}}} 10^{D_{\mathrm{t}}-D_{\mathrm{i}} / 20}+\frac{A_{\mathrm{r}}}{A_{\mathrm{t}}} 10^{D_{\mathrm{t}}-D_{\mathrm{r}} / 20}\right)\left(1-\frac{A_{\mathrm{r}}}{A_{\mathrm{i}}} 10^{D_{\mathrm{i}}-D_{\mathrm{r}} / 20}\right)\right],
$$

where $\alpha$ is attenuation coefficient, $A_{\mathrm{i}}, A_{\mathrm{t}}$ and $A_{\mathrm{r}}$ are the amplitudes of incident wave, transmitted wave and reflected wave respectively, $D_{\mathrm{i}}, D_{\mathrm{t}}, D_{\mathrm{r}}$ are the enlargement indexes of incident wave, transmitted wave and reflected wave respectively, and $h$ is the thickness of samples.

Frequency spectrum analyses were conducted based on the recorded transmitted wave signals using fast Fourier transform (FFT), from which the master frequency can be obtained.

Based on the measured ultrasonic pulse velocities of undamaged and damaged explosive samples, the damage of samples can be calculated as [18]

$$
D=1-\left(C_{p} / C_{p 0}\right)^{2}
$$

where $C_{p 0}$ and $C_{p}$ are the ultrasonic pulse velocities of undamaged and damaged explosive samples, respectively.

\subsection{Density and particle size distribution analysis}

The sample densities before and after impact were measured by using Archimedes method according to the conventional procedure. To quantitatively characterize the variation of explosive particle size distribution induced by impact, a thorough etching technique was developed. A solution for example iso-methyl butyl ketone was selected to dissolve the binder without causing any damage to the explosive crystals. The sample was soaked in the solution for enough time to thoroughly dissolve the binder. After decanting, the explosive powder was collected for particle size distribution analysis. Particle size distribution analysis was carried out on a Malvern2000 
particle sizer, in which distilled water was used as a dispersing medium. Both the undamaged samples and the samples damaged under different impact velocities were measured.

\section{Results and discussion}

Figs. 4-6 show the plan-view images of damaged PBXN-5 impacted at a velocity of $171 \mathrm{~m} / \mathrm{s}$. Fig. 4 is an image of the near field, while Figs. 5 and 6 are the images of mid-field and far-field, respectively, with the projectile incident from right. The micrograph of undamaged PBXN-5 (Fig. 7) is given for comparison. Some randomly distributed microcracks in explosive particles were present in undamaged PBXN-5, which were induced by hot pressing. Though visible macrocracks were not observed in the damaged PBXN-5, a lot of microcracks were present. The density of microcracks in the three characteristic regions were different with the far-field region having the largest number of microcracks. Please note that the substantial preferred orientation of the microcracks and the coalesced larger cracks in damaged PBXN-5 is in the vertical direction.

Image processing was performed for the obtained micrographs to extract the microcracks and then the fractal dimensions were obtained according to the above-mentioned fractal concept. Fractal dimension is directly linked with the extent of microcracking, thus reflects the extent of damage. The fractal dimension of undamaged PBXN-5 was 1.208, which was mainly associated with initial microcracks induced by hot pressing. The fractal dimensions increased to $1.406,1.416$ and 1.450 in near-field, mid-field and far-field regions, respectively, when impacted at a velocity of $171 \mathrm{~m} / \mathrm{s}$, demonstrating that considerable damage was present in impact damaged samples. Impact induced damage was not homogenous in the sample. The far-field region was most seriously damaged. This was consistent with direct visual observation of the micrographs. When the sample was damaged at a higher velocity of $191 \mathrm{~m} / \mathrm{s}$, the fractal dimensions of the three regions increased to $1.602,1.632$ and 1.678, respectively. To correlate fractal dimension and damage, extensive microstructural examination and fractal analysis are needed.

The density variation, ultrasonic velocities, attenuation coefficients, amplitude and master frequency before impact and after impact under different impact loading conditions including

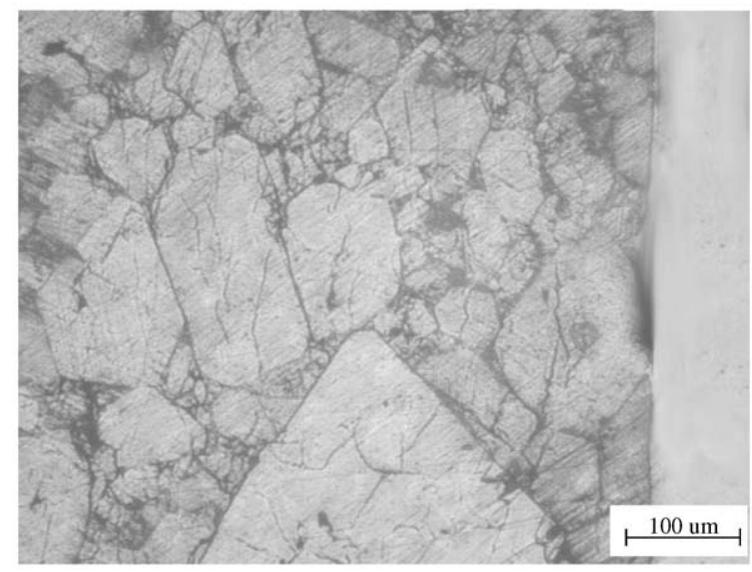

Fig. 4. Damaged PBXN-5, near-field region. 


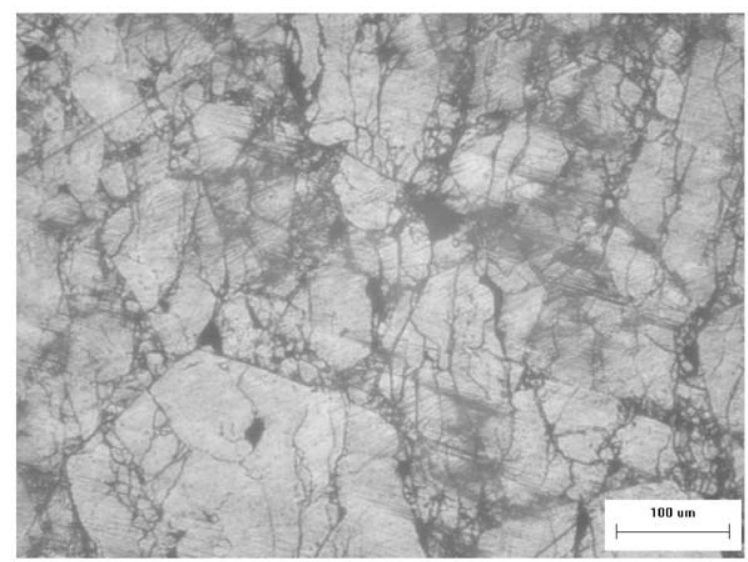

Fig. 5. Damaged PBXN-5, mid-field region.

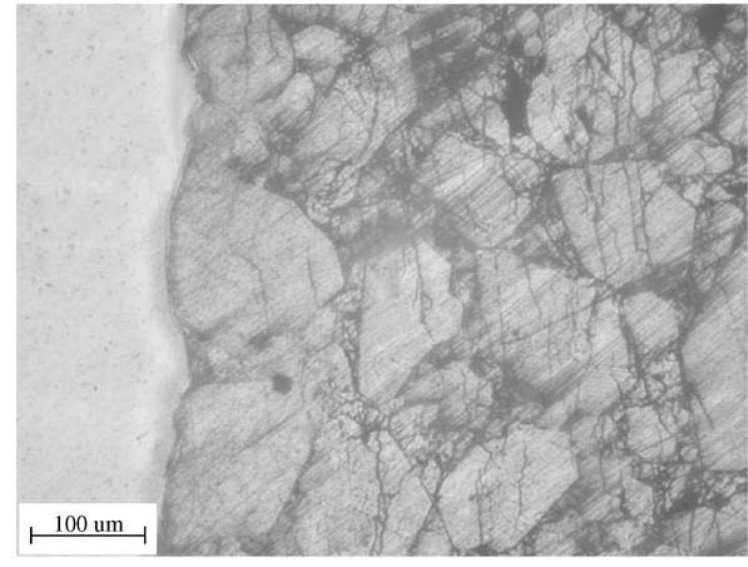

Fig. 6. Damaged PBXN-5, far-field region.

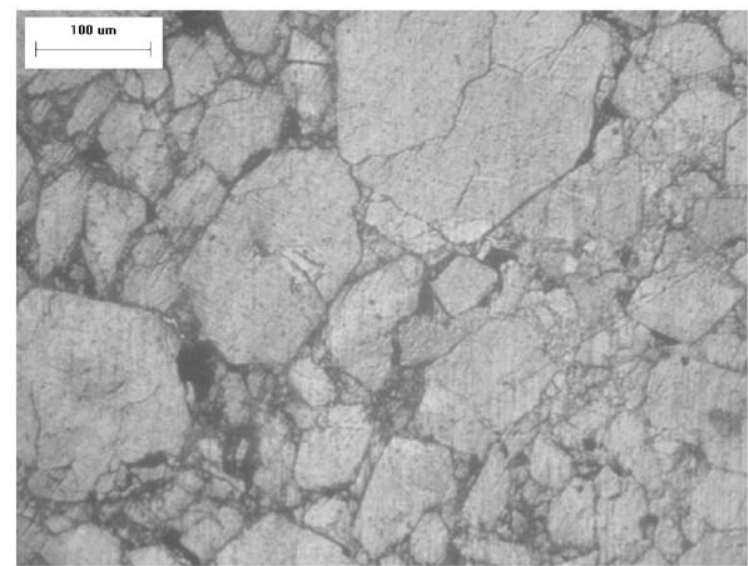

Fig. 7. Plan view of undamaged PBXN-5. 
impact velocities, peak stresses and pulse durations are listed in Table 1. The damage calculated by formula (3) is also listed in Table 1. Due to the presence of extensive microcracks induced by impact, the densities in damaged samples were slightly reduced. The higher the impact velocity was, the more the density reduced. The largest impact velocity used in our experiment was $203.0 \mathrm{~m} / \mathrm{s}$, which correspondingly caused the largest density decrease of $1.32 \%$.

Fig. 8 shows a received transmitted ultrasonic signal of the undamaged sample, while Fig. 9 shows a typical transmitted ultrasonic signal of a damaged sample, demonstrating that the waveforms of transmitted ultrasonic signals are different due to the presence of impact damage. The results show that after impact the ultrasonic velocity decreases and the attenuation coefficient increases due to the presence of impact damage. In general, the higher the impact velocities, the lower the acoustic velocities and the larger the attenuation coefficients. The attenuation coefficient of undamaged PBXN-5 was $2.43 \mathrm{~dB} / \mathrm{mm}$. The attenuation coefficient increased to $2.88 \mathrm{~dB} / \mathrm{mm}$, when the sample was impacted at a velocity of $60.4 \mathrm{~m} / \mathrm{s}$, showing an increase of $18.5 \%$. The attenuation coefficient further increased to $4.72 \mathrm{~dB} / \mathrm{mm}$, when the sample was impacted at a higher velocity of $191.4 \mathrm{~m} / \mathrm{s}$, showing an increase of $94.2 \%$. The presence of impact damage also caused the ultrasonic velocity to decrease. The ultrasonic velocity of undamaged PBXN-5 was $2.10 \mathrm{~km} / \mathrm{s}$. The largest change of ultrasonic velocity in our experiments was $0.45 \mathrm{~km} / \mathrm{s}$, showing a decrease of $21.4 \%$. However correlation between master frequency and impact damage cannot be confirmed according to the data in Table 1. At present, we cannot give further explanation to it. More experiments are planned to do to clarify this issue.

The results of ultrasonic testing also show that attenuation coefficient (amplitude) is more sensitive than ultrasonic velocity. Traditional definition of damage through only acoustic velocities is not accurate enough to assess the damage. Accurate assessment of damage through acoustic parameters should consider amplitude and master frequency in addition to acoustic velocity.

Fig. 10 shows the evolution of HMX particle size under hot pressing and impact loading. The initial particle size distribution of HMX in molding powder is also given for comparison. Hot pressing causes extensive crystal fractures, resulting a decrease of HMX particle size. The particle size of HMX crystals in the initial molding powder was $D[4,3]=139.1 \mu \mathrm{m}$, and $d(0.5)=$ $123.7 \mu \mathrm{m}$, where $D[4,3]$ and $d(0.5)$ refer to volume average particle size and median particle size, respectively. When it was pressed under $200 \mathrm{MPa}$ for $1.5 \mathrm{~h}$, the particle size decreased to $D[4,3]=$ $119.3 \mu \mathrm{m}$, and $d(0.5)=102.3 \mu \mathrm{m}$. Impact loading induced extensive crystal fractures, causing a further decrease of particle size. When the sample was impacted at a velocity of $164.4 \mathrm{~m} / \mathrm{s}$, the particle size decreased to $D[4,3]=89.4 \mu \mathrm{m}$, and $d(0.5)=86.3 \mu \mathrm{m}$. When the sample was impacted at a higher impact velocity of $191.4 \mathrm{~m} / \mathrm{s}$, the particle size further decreased to $D[4,3]=80.5 \mu \mathrm{m}$, and $d(0.5)=78.9 \mu \mathrm{m}$.

Crystal fractures cause an increase of specific area of explosive crystals, which in return affects the reactivity of energetic materials. Impact induced new surfaces from debonding and crystal fractures are vital to assess the reactivity for energetic materials. Our experiments show that impact induced crystal fractures cause the variation of explosive particle size distribution. Demol et al. [19] proposed a reaction model using particle size distribution as a parameter. If the evolution law of particle size distribution under impact loading can be determined, it can be coupled with the damage variable and incorporated with chemical reaction. Then it can be used to simulate the responses of energetic materials to impact loading. Extensive crystal fractures may 
Table 1

Results of ultrasonic measurement

\begin{tabular}{llllllllll}
\hline Samples & $\begin{array}{l}\text { Impact } \\
\text { velocity } \\
\left(\mathrm{m} \mathrm{s}^{-1}\right)\end{array}$ & $\begin{array}{l}\text { Peak stress } \\
(\mathrm{MPa})\end{array}$ & $\begin{array}{l}\text { Pulse duration } \\
(\mathrm{ms})\end{array}$ & $\begin{array}{l}\text { Acoustic } \\
\text { velocity } \\
\left(\mathrm{km} \mathrm{s}^{-1}\right)\end{array}$ & $\begin{array}{l}\text { Attenuation } \\
\text { coefficient } \\
\left(\mathrm{dB} \mathrm{mm}^{-1}\right)\end{array}$ & $\begin{array}{l}\text { Master } \\
\text { frequency } \\
(\mathrm{kHz})\end{array}$ & $\begin{array}{l}\text { Amplitude } \\
A\left(f_{0}\right)\end{array}$ & $\begin{array}{l}\text { Density change } \\
(\%)\end{array}$ & Damage \\
\hline Undamaged & - & - & - & 2.10 & 2.43 & 831 & 0.098 & -1.03 & - \\
126 & 155.0 & 182.9 & 2.55 & 1.76 & 4.57 & 834 & 0.020 & -0.30 \\
128 & 164.4 & 166.0 & 3.87 & 1.70 & 4.63 & 837 & 0.013 & -0.79 & 0.34 \\
129 & 194.1 & NA & NA & 1.68 & 4.53 & 756 & 0.017 & -1.11 & 0.36 \\
130 & 60.4 & 56.3 & 6.71 & 1.74 & 2.88 & 834 & 0.086 & -0.21 & 0.31 \\
131 & 147.8 & NA & NA & 1.70 & 3.98 & 775 & 0.029 & -0.53 & 0.34 \\
132 & 191.4 & 212.5 & 2.85 & 1.65 & 4.72 & 785 & 0.009 & -1.08 & 0.38 \\
143 & 203.0 & 203.5 & 2.94 & 1.73 & 4.65 & 781 & 0.018 & -1.32 & 0.32 \\
\hline
\end{tabular}




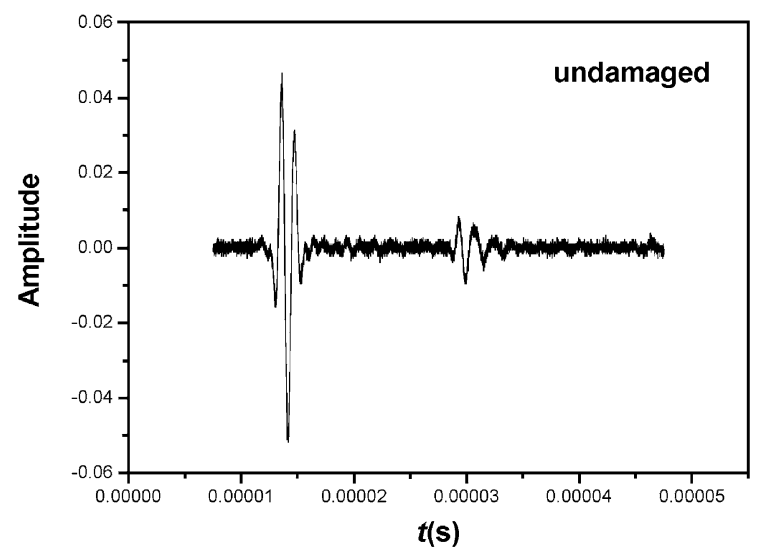

Fig. 8. Transmitted ultrasonic signal of the undamaged sample.

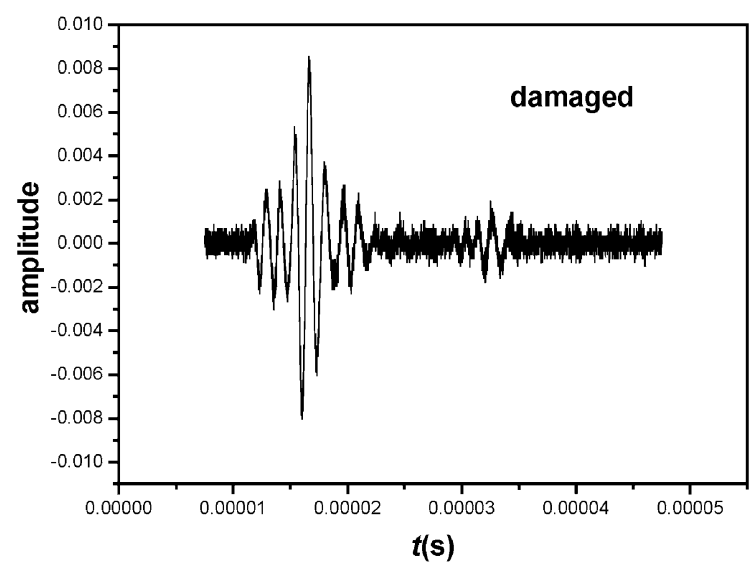

Fig. 9. Transmitted ultrasonic signal of the damaged sample.

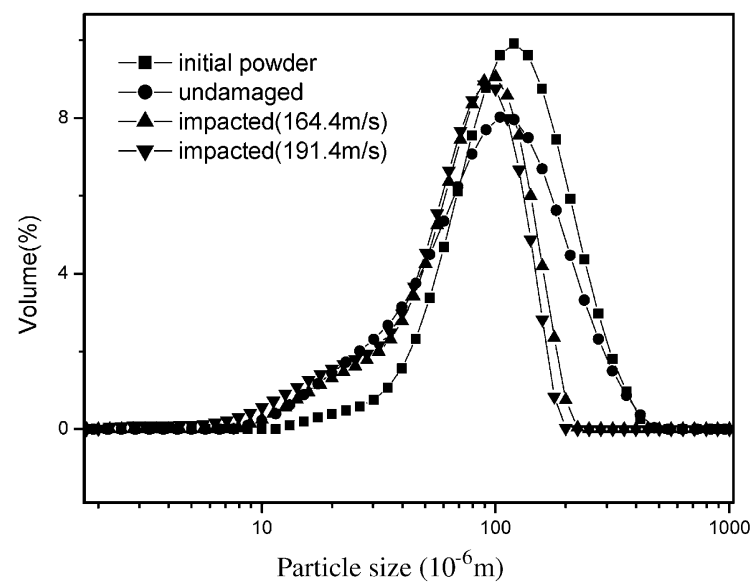

Fig. 10. Particle size distribution before and after impact damage. 
influence the thermal stability, sensitivity and even detonation behavior of energetic materials. This work is currently under way in our laboratory.

\section{Conclusions}

A low-velocity gas gun with a gas buffer can be used to apply long-pulse dynamic loading. Various extents of impact damage can be obtained by adjusting the pressure of the gas buffer and the impact velocities of projectiles. Microscopic examination and fractal analysis of the microstructure reveal that impact damage is not homogeneous. Impact loading induces a lot of microcracks and microvoids, which cause the density to decrease. Ultrasonic testing proves to be an efficient tool to characterize the impact damage of explosive materials. Consistency exists between damage state and ultrasonic parameters including ultrasonic velocities and attenuation coefficients. However, the consistency between damage state and master frequency has not been proved in our experiments. Among the ultrasonic parameters, attenuation coefficient (amplitude) is the most sensitive one. To accurately assess the impact damage, a combination of ultrasonic parameters should be taken into consideration. Due to the large extent of particle-to-particle contact, impact induces considerable crystal fractures, causing the change of particle size distribution. The average particle size decreases with the increase of impact damage.

To deepen our understanding in the impact damage of explosives, a wider range of impact velocities are planned to use. To further investigate impact induced crystal fractures and correlation between impact damage and fractal dimensions and ultrasonic parameters, more experiments need to be done. In addition, considering the characteristics of explosives, special attention should also be paid to the influences of impact damage on the thermal stability and shock sensitivity of explosives.

\section{Acknowledgements}

The authors would like to acknowledge the Chinese National Natural Science Foundation (Grant No. 10002022) for the financial support.

\section{References}

[1] Richter HP, Boyer LR, Graham KJ, Lepie AH, Zwierzchowski NG. Shock sensitivity of damaged energetic materials. In: Lee EL, Short JM, editors. Proceedings of the Ninth Symposium (International) on Detonation. Portland, Oregon; 1989. p. 1295-300.

[2] Sandusky HW, Bernecker RR. Influence of fresh damage on the shock reactivity and sensitivity of several energetic materials. In: Short JM, Tasker DG, editors. Proceedings Tenth International Detonation Symposium. Boston: Massachusetts; 1993. p. 490-8.

[3] Green LG, James E, Lee EL, Chambers ES, Tarver CM, Westmoreland C. Delayed detonation in propellants from low velocity impact. In: Proceedings of Seventh Symposium (International) on Detonation. Naval Surface Weapons Center, White Oak Maryland; 1981. p. 256-64.

[4] Weirich LJ. Shock-wave characterization of energetic booster-rocket propellant WAK-2 and its simulant UGS, SAND89-0917, UC600. 
[5] Demol G, Lambert P, Trumel H. A study of the microstructure of pressed TATB and its evolution after several kinds of solicitations. In: Short JM, Kennedy JE, editors. Papers Summaries-Eleventh International Detonation Symposium. Snowmass; 1998. p. 404-6.

[6] Green L, James E, Lee E. Energetic response of propellants to high velocity impact. In: Short JM, editor. Proceedings of Eighth Symposium (International) on Detonation. New Mexico: Albuquerque; 1985. p. 284-93.

[7] Zhao Feng, Sun Chengwei, Wen Shanggang, Zhao Jianheng, Long Xinping. Brittle fracture of high explosive JO-9159 under plate impact loading. Explosion and Shock Waves 2001;21(2):121-5 [in Chinese].

[8] Sandusky HW, Bernerker RR. Influence of fresh damage on the shock reactivity and sensitivity of several energetic materials. In: Short JM, Tasker DG, editors. Proceedings of Tenth Symposium (International) on Detonation. Boston: Massachusetts; 1993. p. 490-8.

[9] Show YP, Shim VPW. Experimental study of low velocity impact damage in woven fiber composites. J Compos Mater 1998;32:1178-202.

[10] Okafor AC, Otieno AW, Dutta A, Rao VS. Detection and characterization of high-velocity impact damage in advanced composite plates using multi-sensing techniques. Compos Struct 2001;54:289-97.

[11] He Hongliang, Ahrens TJ. Mechanical properties of shock-damaged rocks. Int J Rock Mech Min Sci \& Geomech Abstr 1994;31(5):525-33.

[12] Rafael Colás. On the variation of grain size and fractal dimension in an austenitic stainless steel. Mater Characterization 2001;46:353-8.

[13] Ling Zhong, Bai Yilong. Study on the relation between fractal features of spalling surface and microstructures of an Al-alloy. Explosion and Shock Waves 1994;14(1):2-8 [in Chinese].

[14] Skidmore C, Phillips D, Idar D, Son S. Characterizing the microstructure of selected explosives. LA-UR-99-1201.

[15] Chen Pengwan, Huang Fenglei, Ding Yansheng. An experimental study on the impact damage of explosives. In: Huang Ping, Wang Yajun, Li Shengcai, Qian Xingming, editors. Progress in safety science and technology, vol. III. Beijing: Science Press; 2002. p. 1417-22.

[16] Barnslay M, editor. Fractals everywhere. New York: Academic Press; 1988.

[17] Schmittbuhl J, Vilotte J-P, Roux S. Phys Rev E 1995;51:131.

[18] Cangli Liu, Ahrens TJ. Stress wave attenuation in shock-damaged rocks. Geomechanics Abstracts 1997;5:307.

[19] Demol G, Goutelle JC, Mazel P. CHARME: a reactive model for pressed explosives using pore and grain size distributions as parameters. 1997 APS Topical Conference on Shock Compression of Condensed Matter. p. 353-7. 